\title{
A Direct Method for Calculating Lyapunov Quantities of Two-Dimensional Dynamical Systems
}

\author{
Leonov G.A., Kuznetsov N.V., and Kudryashova E.V. \\ Draft $^{1} 2$
}

\begin{abstract}
A direct method is proposed for studying the behavior of two-dimensional dynamical systems in the critical case when the linear part of the system has two purely imaginary eigenvalues. This method allows one to construct approximations to solutions of the system and to the return time of the trajectory in the form of a finite series in powers of the initial datum. Based on suggested method, computation of Lyapunov quantities in Euclidean coordinates and in the time domain are performed.
\end{abstract}

Keywords: Direct method for computation, Lyapunov quantity, Poincare-Lyapunov constant, focus value, symbolic computations, small limit cycle, 16th Hilbert problem.

The issue of the behavior of a dynamical system when the value of a parameter is close to the boundary of the stability region, which is important for engineering mechanics, involves calculating Lyapunov quantities. According to Bautin's paper [1], "dangerous" and "safe" boundaries are distinguished, a small transgression of which causes small (reversible) or irreversible changes in the state of the system. Such changes correspond, for example, to the scenarios of "soft" and "hard" excitation of oscillations, which were considered by Andronov [2]. In the classical papers by Poincare [3] and Lyapunov [4], for the analysis of the behavior of a system near the stability boundary, the method of calculating the so-called Lyapunov quantities (or Poincare-Lyapunov constants), which determine the behavior of the system near the boundary, was developed. For example, in the case of two complex-conjugate characteristic roots of a two-dimensional system in a neighborhood of a stationary point (a critical case), when the stability boundary is crossed from negative values of the real part of the roots to positive values, if the first nonzero Lyapunov quantity is negative, then there appears a unique stable limit cycle, which contracts to a point under the inverse change of the parameter, which corresponds to a "safe" boundary. On the contrary, if the first nonzero Lyapunov quantity is positive, then, under small changes, the trajectory can diverge infinitely far from the equilibrium state, which corresponds to a "dangerous" boundary. Note also that small disturbances of a system with several zero Lyapunov quantities may lead [5-13] to the appearance of several "small" limit cycles (small oscillations). When calculating Lyapunov quantities, one usually considers the two-dimensional sufficiently smooth system with two purely imaginary eigenvalues of the linear part

$$
\begin{aligned}
& \frac{d x}{d t}=-y+f(x, y), \\
& \frac{d y}{d t}=x+g(x, y) .
\end{aligned}
$$

According to Lyapunov's result [4], problems of a higher dimension (two roots are purely imaginary and the remaining roots are negative) can be reduced to a two-dimensional problem.

While the general expression for the first and second Lyapunov quantities (in terms of expansion coefficients of $f$ and $g$ in the original space) had been computed in the 40-50s of last century [Bautin, 1949; Serebryakova, 1959], the third Lyapunov quantity was computed only in 2008 [Kuznetsov \& Leonov, 2008' Leonov et.

\footnotetext{
${ }^{1}$ Nikolay V. Kuznetsov, nkuznetsov239 at gmail.com (correspondence author)

${ }^{2} \mathrm{PDF}$ slides http://www.math.spbu.ru/user/nk/PDF/Limit-cycles-Focus-values-Lyapunov-quantity-16th-Hilbert.pdf
} 
$a l, 2008]$. Its expression occupies more than four pages and the expression for the fourth Lyapunov quantity occupies more than 45 pages. Therefore the analysis of general expressions of Lyapunov quantities is a difficult algebraic problem.

At present, there exist several methods $[12,13,15,16]$ for finding Lyapunov quantities and their computer implementations, which allow one to find Lyapunov quantities in the form of symbolic expressions that depend on the expansion coefficients of the right-hand sides of the equations of the system. These methods vary in the complexity of the algorithms and the compactness of the produced symbolic expressions. The first method for finding Lyapunov quantities was proposed in [3]. The method is based on the fact that the linear part of the system has the evident integral $x^{2}+y^{2}$, and its idea is to successively construct a time-independent integral for the approximations of the system.

Another approach to calculating Lyapunov quantities involves finding an approximate solution of the system. The classical approach [4] uses changes of variables to reduce the turn-round time of all the trajectories to a constant (as, for example, in the polar coordinate system) as well as procedures for the recursive construction of approximate solutions. In this paper, we propose a direct method for calculating Lyapunov quantities based on the construction of approximate solutions (in the form of a finite sum over the powers of the initial data) in the original Euclidean coordinate system and in a temporal domain. The advantage of this method is its conceptual simplicity and demonstrativeness. This approach can also be applied for the solution of the isochronous center problem, since it allows one to find an approximation to return time of a trajectory depending on the initial data.

\section{Approximation of solutions in Euclidian coordinates}

Following $[14,15]$, we will assume that right-hand sides have continuous $(n+1)$ th-order partial derivatives in an open neighborhood $U$ of radius $R_{U}$ of the point $(x, y)=(0,0)$ :

$$
f(\cdot, \cdot), g(\cdot, \cdot): \mathbb{R} \times \mathbb{R} \rightarrow \mathbb{R} \in \mathbb{C}^{(n+1)}(U) .
$$

Consequently, the following representation is valid in the neighborhood $U$

$$
\begin{aligned}
& f(x, y)=\sum_{k+j=2}^{n} f_{k j} x^{k} y^{j}+o\left((|x|+|y|)^{n}\right)=f_{n}(x, y)+o\left((|x|+|y|)^{n}\right) \\
& g(x, y)=\sum_{k+j=2}^{n} g_{k j} x^{k} y^{j}+o\left((|x|+|y|)^{n}\right)=g_{n}(x, y)+o\left((|x|+|y|)^{n}\right) .
\end{aligned}
$$

The condition of the existence of the $(n+1)$ th partial derivatives with respect to $x$ and $y$ for $f$ and $g$ will be used for the simplicity of presentation; this condition can be weakened.

Consider the solution $x(t, x(0), y(0)), y(t, x(0), y(0))$ of system $(0.1)$ with the initial data

$$
x(0)=0, y(0)=h \quad h \in[0, H],
$$

where $H$ is sufficiently small, and define

$$
x(t, h)=x(t, 0, h), y(t, h)=y(t, 0, h)
$$

1 There exists a positive number $H \in\left(0, R_{U}\right)$, such that, for all $h \in[0, H]$ the solution $(x(t, h), y(t, h))$ is defined for $t \in[0,4 \pi]$.

The validity of the lemma follows from the presence of two purely imaginary eigenvalues of the linear approximation matrix of system (0.1).

Hence, according to $|17|$, the following statement is valid. 
2 If smoothness condition (1.1), is satisfied, then

$$
x(\cdot, \cdot), y(\cdot, \cdot) \in \mathbb{C}^{(n+1)}([0,4 \pi] \times[0, H]) .
$$

In what follows, we will use the smoothness of the functions $f$ and $g$ and follow the first Lyapunov method on a finite time interval [18-19].

Let us introduce the notation $\widetilde{x}_{h^{k}}(t)=\left.\frac{\partial^{k} x(t, \eta)}{k ! \partial^{k} \eta}\right|_{\eta=0}, \widetilde{y}_{h^{k}}(t)=\left.\frac{\partial^{k} y(t, \eta)}{k ! \partial^{k} \eta}\right|_{\eta=0}$. The sums

$$
x_{h^{m}}(t, h)=\sum_{k=1}^{m} \widetilde{x}_{h^{k}}(t) h^{k}, \quad y_{h^{m}}(t, h)=\sum_{k=1}^{m} \widetilde{y}_{h^{k}}(t) h^{k}
$$

will be called the $m$ th approximation to the solution of the system with respect to $h$.

The condition that the right-hand sides of the system are smooth implies that, for every fixed $t$ the solution of the system can be represented in the form

$$
x(t, h)=x_{h^{m}}(t, h)+o\left(h^{m}\right), \quad y(t, h)=y_{h^{m}}(t, h)+o\left(h^{m}\right),
$$

where the remainder terms $o\left(h^{m}\right)$ belong to $\mathbb{C}^{1}$ and are uniformly bounded with respect to time on the set $([0,4 \pi] \times[0, H])$.

Let us substitute representation (1.5) for $m=1$ into system (0.1). In the obtained equalities, we equate the coefficients at $h^{1}$ and, taking into account ( (1.2), obtain

$$
\frac{d \widetilde{x}_{h^{1}}(t)}{d t}=-\widetilde{y}_{h^{1}}(t), \quad \frac{d \widetilde{y}_{h^{1}}(t)}{d t}=\widetilde{x}_{h^{1}}(t)
$$

Hence, taking into account conditions (1.3), for the initial data, we get the following relations for the first approximation with respect to $h$ of the solution $(x(t, h), y(t, h))$ :

$$
x_{h^{1}}(t, h)=\widetilde{x}_{h^{1}}(t) h=-h \sin (t), y_{h^{1}}(t, h)=\widetilde{y}_{h^{1}}(t) h=h \cos (t) .
$$

Similarly, to find the second approximation $\left(x_{h^{2}}(t, h), y_{h^{2}}(t, h)\right)$ we substitute the representation

$$
x(t, h)=x_{h^{2}}(t, h)+o\left(h^{2}\right), \quad y(t, h)=y_{h^{2}}(t, h)+o\left(h^{2}\right)
$$

into formula (1.2) for $f(x, y)$ and $g(x, y)$. Note that, in view of (1.2), the coefficients at $h^{2}$ in the obtained expressions for $f$ and $g$ (let us denote them by $u_{h^{2}}^{f}$ and $u_{h^{2}}^{g}$ respectively) depend on $\widetilde{x}_{h^{1}}(t)$ and $\widetilde{y}_{h^{1}}(t)$, only; i.e., by virtue of (1.7), they are known functions of time and are independent of the unknown functions $\widetilde{x}_{h^{2}}(t)$ and $\widetilde{y}_{h^{2}}(t)$. Thus,

$$
\begin{aligned}
& f\left(x_{h^{2}}(t, h)+o\left(h^{2}\right), y_{h^{2}}(t, h)+o\left(h^{2}\right)\right)=u_{h^{2}}^{f}(t) h^{2}+o\left(h^{2}\right), \\
& g\left(x_{h^{2}}(t, h)+o\left(h^{2}\right), y_{h^{2}}(t, h)+o\left(h^{2}\right)\right)=u_{h^{2}}^{g}(t) h^{2}+o\left(h^{2}\right) .
\end{aligned}
$$

In order to find $\widetilde{x}_{h^{2}}(t)$ and $\widetilde{y}_{h^{2}}(t)$, we substitute (1.8) into system (0.1) and get

$$
\frac{d \widetilde{x}_{h^{2}}(t)}{d t}=-\widetilde{y}_{h^{2}}(t)+u_{h^{2}}^{f}(t), \quad \frac{d \widetilde{y}_{h^{2}}(t)}{d t}=\widetilde{x}_{h^{2}}(t)+u_{h^{2}}^{g}(t)
$$

3 For the solutions of the system

$$
\frac{d \widetilde{x}_{h^{k}}(t)}{d t}=-\widetilde{y}_{h^{k}}(t)+u_{h^{k}}^{f}(t), \quad \frac{d \widetilde{y}_{h^{k}}(t)}{d t}=\widetilde{x}_{h^{k}}(t)+u_{h^{k}}^{g}(t)
$$

with the initial data

$$
\widetilde{x}_{h^{k}}(0)=0, \quad \widetilde{y}_{h^{k}}(0)=0
$$


we have

$$
\begin{aligned}
& \widetilde{x}_{h^{k}}(t)=u_{h^{k}}^{g}(0) \cos (t)+\cos (t) \int_{0}^{t} \cos (\tau)\left(\left(u_{h^{k}}^{g}(\tau)\right)^{\prime}+u_{h^{k}}^{f}(\tau)\right) \mathrm{d} \tau+ \\
& +\sin (t) \int_{0}^{t} \sin (\tau)\left(\left(u_{h^{k}}^{g}(\tau)\right)^{\prime}+u_{h^{k}}^{f}(\tau)\right) \mathrm{d} \tau-u_{h^{k}}^{g}(t), \\
& \widetilde{y}_{h^{k}}(t)=u_{h^{k}}^{g}(0) \sin (t)+\sin (t) \int_{0}^{t} \cos (\tau)\left(\left(u_{h^{k}}^{g}(\tau)\right)^{\prime}+u_{h^{k}}^{f}(\tau)\right) \mathrm{d} \tau- \\
& -\cos (t) \int_{0}^{t} \sin (\tau)\left(\left(u_{h^{k}}^{g}(\tau)\right)^{\prime}+u_{h^{k}}^{f}(\tau)\right) \mathrm{d} \tau .
\end{aligned}
$$

Equalities (1.12) are verified immediately by differentiation.

Repeating the specified procedure of determining the coefficients $\widetilde{x}_{h^{k}}$ and $\widetilde{y}_{h^{k}}$, and the functions $u_{h^{k}}^{f}(t)$ and $u_{h^{k}}^{g}(t)$, we find successively by formula (1.12) the approximations $\left(x_{h^{k}}(t, h), y_{h^{k}}(t, h)\right)$ for $k=1, \ldots, n$. For $h \in[0, H]$ and $t \in[0,4 \pi]$ we have

$$
\begin{aligned}
& x(t, h)=x_{h^{n}}(t, h)+o\left(h^{n}\right)=\sum_{k=1}^{n} \widetilde{x}_{h^{k}}(t) h^{k}+o\left(h^{n}\right), \\
& y(t, h)=y_{h^{n}}(t, h)+o\left(h^{n}\right)=\sum_{k=1}^{n} \widetilde{y}_{h^{k}}(t) h^{k}+o\left(h^{n}\right) .
\end{aligned}
$$

Here, by Lemma 2,

$$
\widetilde{x}_{h^{k}}(\cdot), \widetilde{y}_{h^{k}}(\cdot) \in \mathbb{C}^{n}([0,4 \pi]), \quad k=1, \ldots, n
$$

and the estimate $o\left(h^{n}\right)$ is uniform $\forall t \in[0,4 \pi]$. From (1.11) and the choice of the initial data in (1.6) we obtain

$$
x_{h^{k}}(0, h)=x(0, h)=0, \quad y_{h^{k}}(0, h)=y(0, h)=h, \quad k=1, \ldots, n .
$$

\section{Computation of Lyapunov quantities in the time domain}

Following $|20|$, we consider for the initial datum $h \in(0, H]$, the time $T(h)$ of the first intersection of the solution $(x(t, h), y(t, h))$ with the half-line $\{x=0, y>0\}$. Define (by continuity) the function $T(h)$ at zero: $T(0)=2 \pi$. According to (1.7), the first approximation of the solution intersects the half-line in time $2 \pi$, hence, the intersection time can be represented in the form

$$
T(h)=2 \pi+\Delta T(h),
$$

where $\Delta T(h)=O(h)$. By the definition of $T(h)$, we have

$$
x(T(h), h)=0 .
$$

According to (1.4), the function $x(\cdot, \cdot)$ has continuous partial derivatives with respect to both arguments up to the order $n$ and $\dot{x}(t, h)=-\cos (t) h+o(h))$, therefore, by the implicit function theorem, $T(\cdot)$ is $n$-times differentiable. One can show that $T(h)$ is also $n$-times differentiable at zero. Then,

$$
T(h)=2 \pi+\sum_{k=1}^{n} \widetilde{T}_{k} h^{k}+o\left(h^{n}\right), \quad \widetilde{T}_{k}=\frac{1}{k !} \frac{d^{k} T(h)}{d h^{k}}
$$

Substituting expression (2.2) for $t=T(h)$ into the right-hand side of the first equation in (1.13) and denoting the coefficient at $h^{k}$ by $\widetilde{x}_{k}$, we obtain the expansion of $x(T(h), h)$ in powers of $h$

$$
x(T(h), h)=\sum_{k=1}^{n} \widetilde{x}_{k} h^{k}+o\left(h^{n}\right) .
$$


In order to express the coefficients $\widetilde{x}_{k}$ in terms of the coefficients $\widetilde{T}_{k}$ we set $t=2 \pi+\tau$ in $(1.13)$

$$
x(2 \pi+\tau, h)=\sum_{k=1}^{n} \widetilde{x}_{h^{k}}(2 \pi+\tau) h^{k}+o\left(h^{n}\right) .
$$

By smoothness condition (1.14)), we have

$$
\widetilde{x}_{h^{k}}(2 \pi+\tau)=\widetilde{x}_{h^{k}}(2 \pi)+\sum_{m=1}^{n} \widetilde{x}_{h^{k}}^{(m)}(2 \pi) \frac{\tau^{m}}{m !}+o\left(\tau^{n}\right), \quad k=1, \ldots, n .
$$

Let us substitute the obtained representation into expression (2.4) for the solution $x(2 \pi+\tau, h)$ for $\tau=\Delta T(h)$ and gather coefficients at equal powers of $h$. Since $(\Delta T(h))^{n}=O\left(h^{n}\right)$, we find in view of expression (2.2) for $T(h)$, and according to $(2.1)$ that

$$
\begin{array}{ll}
h: & 0=\widetilde{x}_{1}=\widetilde{x}_{h^{1}}(2 \pi) \\
h^{2}: & 0=\widetilde{x}_{2}=\widetilde{x}_{h^{2}}(2 \pi)+\widetilde{x}_{h^{1}}^{\prime}(2 \pi) \widetilde{T}_{1} \\
h^{3}: & 0=\widetilde{x}_{3}=\widetilde{x}_{h^{3}}(2 \pi)+\widetilde{x}_{h^{1}}^{\prime}(2 \pi) \widetilde{T}_{2}+\frac{1}{2} \widetilde{x}_{h^{2}}^{\prime}(2 \pi) \widetilde{T}_{1}+\frac{1}{2} \widetilde{x}_{h^{1}}^{\prime \prime}(2 \pi) \widetilde{T}_{1}^{2}, \\
\ldots & \\
h^{n}: & 0=\widetilde{x}_{n}=\widetilde{x}_{h^{n}}(2 \pi)+\widetilde{x}_{h^{1}}^{\prime}(2 \pi) \widetilde{T}_{n-1}+\ldots
\end{array}
$$

From these relations, we can successively find the coefficients $\widetilde{T}_{k=1, \ldots, n-1}$, because the expression for $\widetilde{x}_{k}$ may contain the coefficients $\widetilde{T}_{1 \leqslant m<k}$ only and the factor $\widetilde{x}_{h^{1}}^{\prime}(2 \pi)$ at $\widetilde{T}_{k-1}$ is equal to -1 .

Let us proceed similarly to determine the coefficients $\widetilde{y}_{k}$ of the expansion

$$
y(T(h), h)=\sum_{k=1}^{n} \widetilde{y}_{k} h^{k}+o\left(h^{n}\right) .
$$

Substitute the representation

$$
\widetilde{y}_{h^{k}}(2 \pi+\Delta T(h))=\widetilde{y}_{h^{k}}(2 \pi)+\sum_{m=1}^{n} \widetilde{y}_{h^{k}}^{(m)}(2 \pi) \frac{\Delta T(h)^{m}}{m !}+o\left(h^{n}\right), \quad k=1, \ldots, n
$$

into the expression

$$
y(2 \pi+\Delta T(h), h)=\sum_{k=1}^{n} \widetilde{y}_{h^{k}}(2 \pi+\Delta T(h)) h^{k}+o\left(h^{n}\right) .
$$

Equating coefficients at equal powers of $h$, we get the equalities

$$
\begin{array}{ll}
h: & \widetilde{y}_{1}=\widetilde{y}_{h^{1}}(2 \pi), \\
h^{2}: & \widetilde{y}_{2}=\widetilde{y}_{h^{2}}(2 \pi)+\widetilde{y}_{h^{1}}^{\prime}(2 \pi) \widetilde{T}_{1}, \\
h^{3}: & \widetilde{y}_{3}=\widetilde{y}_{h^{3}}(2 \pi)+\widetilde{y}_{h^{1}}^{\prime}(2 \pi) \widetilde{T}_{2},+\frac{1}{2} \widetilde{y}_{h^{2}}^{\prime}(2 \pi) \widetilde{T}_{1}+\frac{1}{2} \widetilde{y}_{h^{1}}^{\prime \prime}(2 \pi) \widetilde{T}_{1}^{2}, \\
\cdots & \\
h^{n}: & \widetilde{y}_{n}=\widetilde{y}_{h^{n}}(2 \pi)+\widetilde{y}_{h^{1}}^{\prime}(2 \pi) \widetilde{T}_{n-1}+\ldots
\end{array}
$$

which can be used to successively find $\widetilde{y}_{k=1, \ldots, n}$, where the values $\widetilde{y}_{h^{k=1, . ., n}}(\cdot) \widetilde{T}_{k=1, . ., n-1}$ were defined above.

Thus, for $n=2 m+1$ and under the condition $f(\cdot, \cdot), g(\cdot, \cdot) \in \mathbb{C}^{(2 m+2)}(U)$ we have successively found approximations of the solution $(x(t, h), y(t, h))$ at the time $t=T(h)$ of the first intersection with the half-line $\{x=0, y>0\}$ accurate to $o\left(h^{2 m+1}\right)$ as well as an approximation of the time $T(h)$ itself accurate to $o\left(h^{2 m}\right)$. If $\widetilde{y}_{k}=0$ for $k=2, . ., 2 m$, then $\widetilde{y}_{2 m+1}$ is called the $m$ th Lyapunov quantity $\mathrm{L}_{m}$. Note that, according to Lyapunov's theorem, the index $k$ of the first nonzero coefficient of the expansion $\widetilde{y}_{k}$ is always odd, and the sign of $\widetilde{y}_{k}$ (the Lyapunov quantity) for sufficiently small initial data $h$ determines the qualitative behavior (twisting, untwisting) of the trajectory $(x(t, h), y(t, h))$ on the plane. 


\section{Solution approximations and Lyapunov quantities for the Liénard system}

Limit cycles of two-dimensional systems can often be investigated by reduction to the Liénard system of a special form $|21|$. For example, in the case of quadratic systems, such a reduction $|22|$ allows one to asymptotically integrate the system and obtain analytic conditions for the existence of "large" limit cycles |23|.

Using the methods considered above, we find approximations of a solution of the Liénard system

$$
\begin{aligned}
& \dot{x}=-y, \\
& \dot{y}=x+g_{x 1}(x) y+g_{x 0}(x)
\end{aligned}
$$

Here $g_{x 1}(x)=g_{11} x+g_{21} x^{2} \ldots, g_{x 0}(x)=g_{20} x^{2}+g_{30} x^{2} \ldots$.

Then, for the approximation of the solution, we have

$\widetilde{x}_{h^{1}}(t)=-\sin (t)$

$\widetilde{x}_{h^{2}}(t)=\frac{1}{3}\left(-g_{11} \sin (t) \cos (t)+\sin (t) g_{11}-g_{20} \cos (t)^{2}+2 \cos (t) g_{20}-g_{20}\right)$

$\widetilde{x}_{h^{3}}(t)=\frac{1}{72}\left(-9 \sin (t) g_{11}^{2} \cos (t)^{2}+9 g_{30} \sin (t) \cos (t)^{2}+6 \sin (t) \cos (t)^{2} g_{20}^{2}+16 g_{11}^{2} \sin (t) \cos (t)-32 \sin (t) \cos (t) g_{20}^{2}-\right.$ $4 \sin (t) g_{20}^{2}-9 \sin (t) g_{21} t+9 \sin (t) g_{11} g_{20} t-10 \sin (t) g_{11}^{2}+18 \sin (t) g_{30}+48 g_{11} g_{20} \cos (t)^{2}+30 \cos (t) g_{20}^{2} t+3 \cos (t) g_{11}^{2} t-$ $\left.15 g_{11} g_{20} \cos (t)^{3}-33 g_{11} \cos (t) g_{20}-27 \cos (t) g_{30} t+9 \cos (t) g_{21}-9 g_{21} \cos (t)^{3}\right)$

$$
\begin{aligned}
& \widetilde{y}_{h^{1}}(t)=\cos (t) \\
& \widetilde{y}_{h^{2}}(t)=\frac{1}{3}\left(-2 g_{20} \sin (t) \cos (t)+2 \sin (t) g_{20}-g_{11}+2 g_{11} \cos (t)^{2}-\cos (t) g_{11}\right) \\
& \widetilde{y}_{h^{3}}(t)=\frac{1}{72}\left(-27 g_{21} \cos (t)^{2} \sin (t)+18 \sin (t) g_{21}+30 \sin (t) g_{20}^{2} t-27 \sin (t) g_{30} t+3 \sin (t) g_{11}^{2} t+96 g_{11} g_{20} \cos (t) \sin (t)-\right.
\end{aligned}
$$
$42 \sin (t) g_{11} g_{20}-45 \sin (t) g_{11} g_{20} \cos (t)^{2}-11 g_{11}^{2} \cos (t)-14 \cos (t) g_{20}^{2}+27 g_{30} \cos (t)-18 \cos (t)^{3} g_{20}^{2}+27 g_{11}^{2} \cos (t)^{3}-$ $\left.27 g_{30} \cos (t)^{3}+16 g_{11}^{2}-9 \cos (t) g_{11} g_{20} t+64 \cos (t)^{2} g_{20}^{2}+9 \cos (t) g_{21} t-32 g_{11}^{2} \cos (t)^{2}-32 g_{20}^{2}\right)$

For the first three Lyapunov quantities, we have

$$
\mathrm{L}_{1}=-\frac{\pi}{4}\left(g_{20} g_{11}-g_{21}\right) .
$$

If $g_{21}=g_{20} g_{11}$, then $\mathrm{L}_{1}=0$ and

$$
\mathrm{L}_{2}=\frac{\pi}{24}\left(3 g_{41}-5 g_{20} g_{31}-3 g_{40} g_{11}+5 g_{20} g_{30} g_{11}\right) .
$$

If $g_{41}=\frac{5}{3} g_{20} g_{31}+g_{40} g_{11}-\frac{5}{3} g_{20} g_{30} g_{11}$, then $\mathrm{L}_{2}=0$ and

$$
\begin{aligned}
\mathrm{L}_{3}= & -\frac{\pi}{576}\left(70 g_{20}^{3} g_{30} g_{11}+105 g_{20} g_{51}+105 g_{30}^{2} g_{11} g_{20}+63 g_{40} g_{31}\right. \\
& \left.-63 g_{11} g_{40} g_{30}-105 g_{30} g_{31} g_{20}-70 g_{20}^{3} g_{31}-45 g_{61}-105 g_{50} g_{11} g_{20}+45 g_{60} g_{11}\right) .
\end{aligned}
$$

\section{Lyapunov quantities and time constants for Duffing equation}

Consider the Duffing equation represented as the system

$$
\dot{x}=-y, \dot{y}=x+x^{3} .
$$

For $x_{0}=0, y_{0}=h_{y}$ we have

$$
y(t)^{2}+x(t)^{2}+\frac{1}{2} x(t)^{4}=h_{y}^{2} .
$$


For the crossing time $T\left(h_{y}\right)$ from $d t / d y=\left(x+x^{3}\right)^{-1}$ we get

$$
\begin{aligned}
T\left(h_{y}\right) & =4 \int_{0}^{h_{y}} \frac{\mathrm{d} y}{\sqrt{-1+\sqrt{1+2 h_{y}^{2}-2 y^{2}}} \sqrt{1+2 h_{y}^{2}-2 y^{2}}} \\
& =\int_{0}^{\pi / 2} \frac{-h_{y} \sin (z) \mathrm{d} z}{\sqrt{-1+\sqrt{1+2 h_{y}^{2} \sin ^{2} z}} \sqrt{1+2 h_{y}^{2} \sin ^{2} z}}= \\
& =2 \pi-\frac{3 \pi}{4} h_{y}^{2}+\frac{105 \pi}{128} h_{y}^{4}-\frac{1155 \pi}{1024} h_{y}^{6}+o\left(h_{y}^{6}\right) .
\end{aligned}
$$

Below, we represent a list of the obtained approximations of solution:

$$
\begin{aligned}
& \widetilde{x}_{h^{1}}(t)=-\sin (t), \widetilde{y}_{h^{1}}(t)=\cos (t) ; \quad \widetilde{x}_{h^{2}}(t)=\widetilde{y}_{h^{2}}(t)=0 ; \\
& \widetilde{x}_{h^{3}}(t)=\frac{1}{8} \cos (t)^{2} \sin (t)-\frac{3}{8} t \cos (t)+\frac{1}{4} \sin (t), \\
& \widetilde{y}_{h^{3}}(t)=-\frac{3}{8} t \sin (t)+\frac{3}{8} \cos (t)-\frac{3}{8} \cos (t)^{3}
\end{aligned}
$$

Here the Lyapunov quantities are equal to zero by virtue of (4.3), and the periodic solution is approximated by a series with nonperiodic coefficients.

\section{Example of algorithm realization in Matlab}

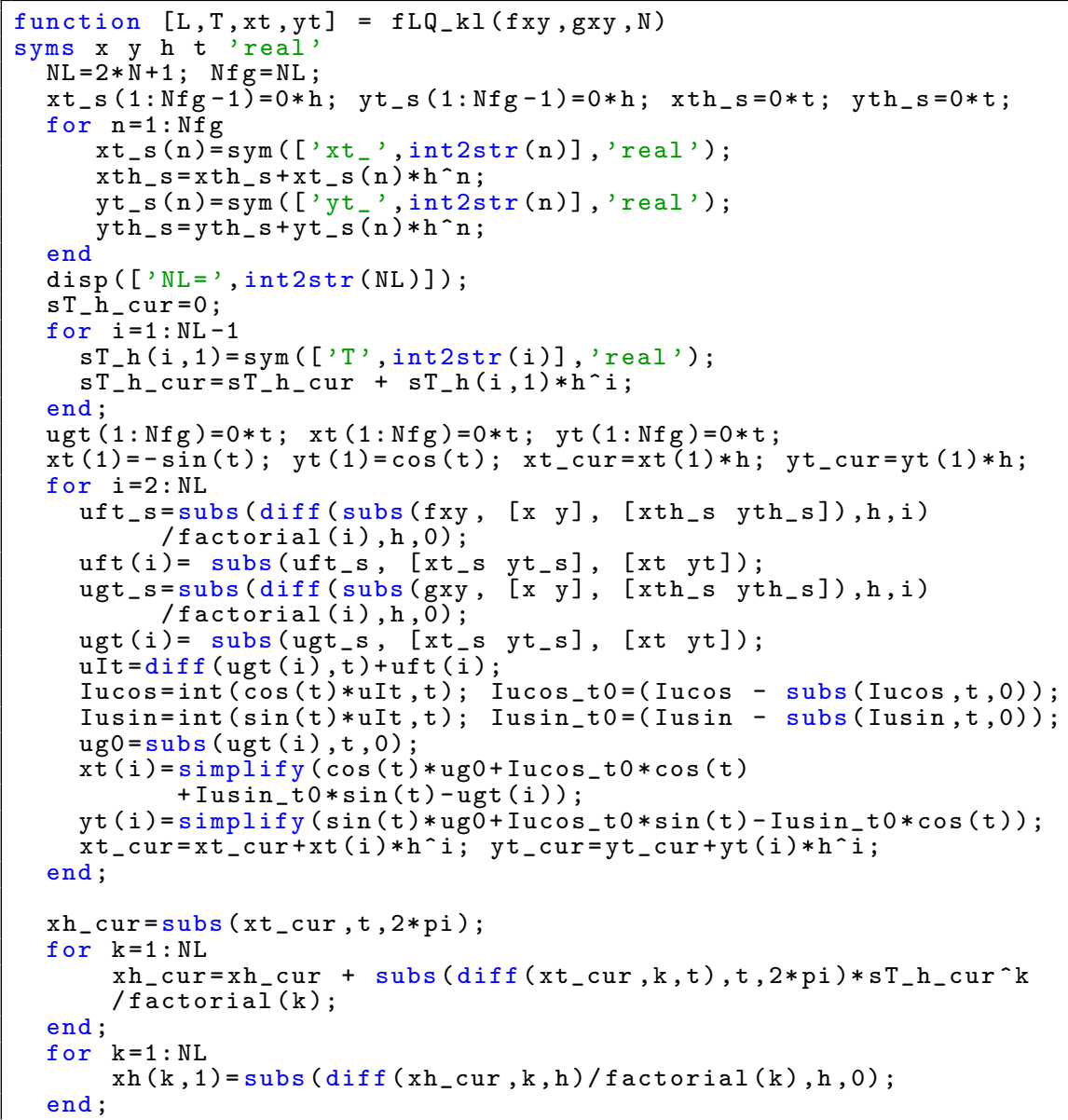




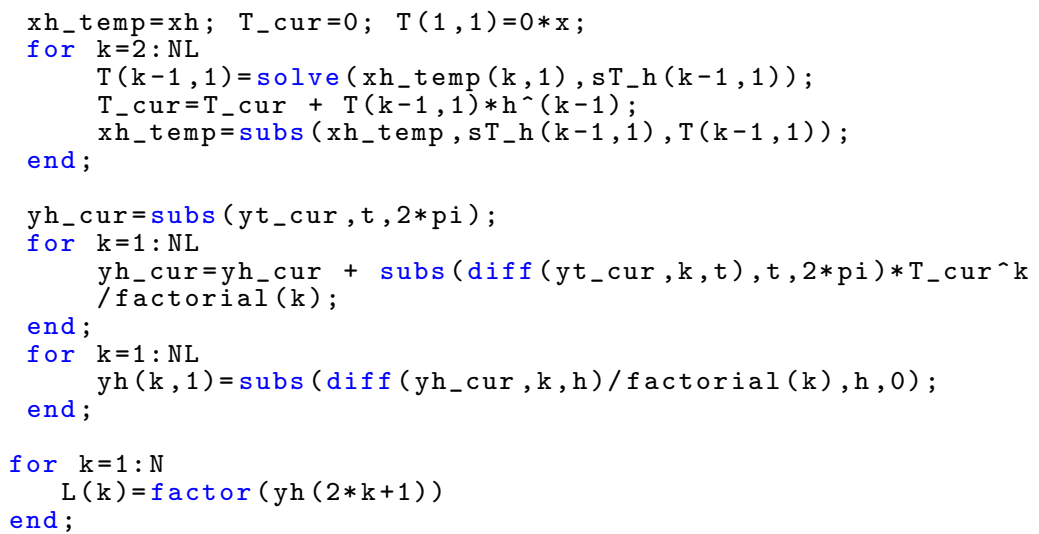

Computation of Lyapunov quantities for Lienard equation in Matlab

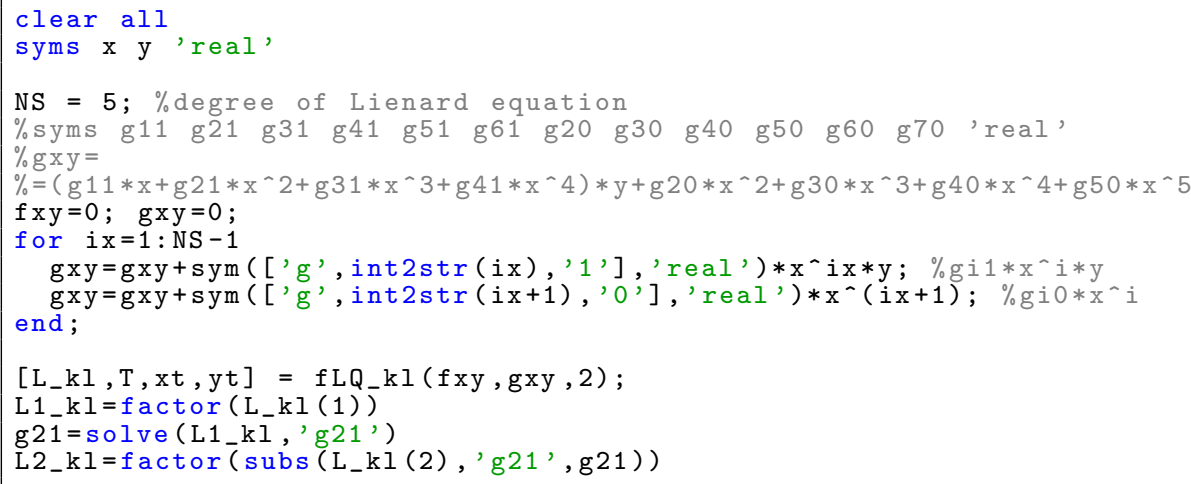

Computation of Lyapunov quantities and time constants for Duffing equation in Matlab

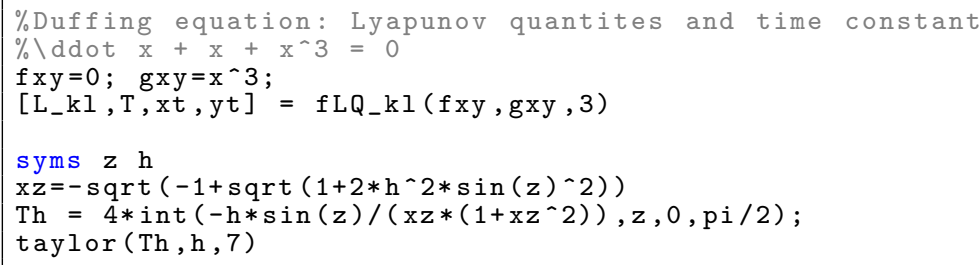

\section{References}

[1] N. N. Bautin, Behavior of Dynamical Systems Near the Boundaries of the Stability Region (Gostekhizdat, Leningrad, 1949) [in Russian].

[2] A. A. Andronov, A. A. Vitt, and S. E. Khaikin, Theory of Oscillations (Fizmatgiz, Moscow, 1959; Pergamon, Oxford, 1966).

[3] H. Poincaré, J. Math. Pures Appl. 1 (4), 167 (1885).

[4] A. M. Lyapunov, The General Problem of the Stability of Motion (Izd. Kharkovsk. Mat. O-va, Kharkov, 1892; Taylor \& Francis, London, 1992).

[5] N. N. Bautin, Mat. Sb. 30 (72) (1), 181 [Amer. Math. Soc. Transl. 100, 1 (1954)].

[6] N. G. Lloyd, in New Directions in Dynamical systems (Cambridge Univ. Press, Cambridge, 1988), Ser. London Math. Soc. Lecture Note, Vol. 127 , pp. 192-234.

[7] J. Li, Internat. J. Bifurcation Chaos 13 (1), 47 (2003). 
[8] Chavarriga J., Grau M. Some open problems related to 16th Hilbert problem // Sci. Ser. A Math. Sci. N.S., 2003. 9. P. 1-26.

[9] Lynch S. Symbolic computation of Lyapunov quantities and the second part of Hilbert's sixteenth problem. Chapter in the book Differential Equations with Symbolic Computations, 2005. P. 1-26.

[10] Dumortier F., Llibre J., Artes J. Qualitative Theory of Planar Differential Systems. Berlin: Springer, 2006.

[11] Christopher C. \& Li Ch. Limit cycles of differential equations. Advanced Courses in Mathematics. CRM Barcelona. Basel: Birkhauser Verlag, 2007.

[12] Gine J. On some problems in planar differential systems and Hilbert's 16th problem // Chaos, Solutions and Fractals. 2007. Vol. 31. P. 1118-1134.

[13] Yu P., Chen G. Computation of focus values with applications // Nonlinear Dynamics. 2008. 51(3).

[14] G. A. Leonov, N. V. Kuznetsov, and E. V. Kudryashova, Cycles of two-dimensional systems: Computer calculations, proofs, and experiments, Vestnik St. Petersburg University: Mathematics 41 (3) , pp. 216250 (2008) (doi:10.3103/S1063454108030047).

[15] N. V. Kuznetsov, G. A. Leonov, Lyapunov quantities, limit cycles and strange behavior of trajectories in two-dimensional quadratic systems, Journal of Vibroengineering 10 (4), 460 (2008).

[16] N. V. Kuznetsov, Stability and Oscillations of Dynamical Systems: Theory and Applications (Jyväskylä Univ., Jyväskylä, 2008).

[17] P. Hartman, Ordinary Diffierential Equations (Wiley, New York, 1964).

[18] S. Lefschetz, Diffierential Equations: Geometric Theory (Interscience, New York, 1957).

[19] L. Cesari, Asymptotic Behavior and Stability Problems in Ordinary Diffierential Equations (SpringerVerlag, Berlin, 1959).

[20] I. G. Malkin, Theory of Stability of Motion (Nauka, Moscow, 1966) [in Russian].

[21] L. A. Cherkass, Diffierents. Uravneniya 9 (3), 1422 (1973).

[22] G. A. Leonov, Diffierential Equations Dynam. Systems 5 (3-4), 289 (1997).

[23] G. A. Leonov, Dokl. Ross. Akad. Nauk, Ser. Mekh. 426 (1), 47 (2009).

[24] N. V. Kuznetsov, G. A. Leonov, Computation of the first Lyapunov quantity for the second-order dynamical system, IFAC Proceedings Volumes (IFAC-PapersOnline) 3 (PART 1), pp. 87-89 (2007) (doi:10.3182/20070829-3-RU-4912.00014) 